\title{
Determination of Postural Control Mechanism in Overweight Adults Using The Artificial Neural Networks System and Nonlinear Autoregressive Moving Average Model
}

\author{
Thunyanoot PrasertsaKuL ${ }^{*}$, Warakorn CharoensuK ${ }^{*}$, \#
}

\begin{abstract}
Being overweight is one of several causes of balance impairment, and it increases the risk of falls. Balance assessments help diagnose this impairment. The outcomes from these assessments are not usually clear to investigate balance impairment in overweight adults. Several methods such as mathematical modeling can be used to investigate the postural control mechanisms in normal balance function. However, there is no study that is focused on the postural control mechanisms in overweight adults. This study aimed to define the postural control models underlying the application of the artificial neural network (ANN) systems in normal weight and overweight populations. Ten participants were recruited and separated into two groups: normal weight (NW) and overweight (OW). There were two processes for determining the postural model in both groups. First, the optimal orders of the nonlinear autoregressive moving average (NARMA) model and the hidden nodes of the ANN system were identified. Mean square error (MSE), Akaike's information criteria (AIC) and residual variance (RV) were used to identify these variables for both groups. Second, the coefficients of these models were defined by the learned weights in the ANN system. The MSE, percent coefficient of variation $(\% \mathrm{CV})$, Kolmogorov-Smirnov (KS) test and maximal distance of cumulative distribution function (CDF) were defined to evaluate the performance of the postural models. Furthermore, the orders of the NARMA model and relative importance were utilized to distinguish the postural control mechanisms between the two groups. During the training process, our results indicated that low MSE, AIC and RV were the criteria for hidden nodes and order selection in the NARMA model, which resulted in different patterns of postural models in each group. In the case of the testing process, the findings revealed that the proposed technique could present different postural control strategies for each group. The findings indicated that the postural control mechanism of NW subjects relied on the center of pressure (CoP) in the anterior-posterior (AP) direction, while body sway in the medio-lateral (ML) direction was vital to maintain equilibrium in the OW subjects. Accordingly, the proposed technique could be used to investigate the difference in postural control mechanism between the two groups.
\end{abstract}

Keywords: autoregressive moving average, neural network, nonlinear time series model, postural control, overweight.

Adv Biomed Eng. 9: pp. 154-166, 2020.

Received on October 14, 2019; revised on March 10, 2020; accepted on May 17, 2020.

* Department of Biomedical Engineering, Faculty of Engineering, Mahidol University, Nakorn Phathom, Thailand.

\# SMART Motion Analysis and Rehabilitation Technology Laboratory, Department of Biomedical Engineering, Faculty of Engineering, Mahidol University, 999 Phuttamonthon Rd. 4, Salaya, Phuttamonthon, Nakhon Pathom 73170, Thailand.

E-mail: warakorn.cha@mahidol.ac.th access article distributed under the terms of the Creative Commons BY 4.0 International (Attribution) License (https:// creativecommons.org/licenses/by/4.0/legalcode), which permits the unrestricted distribution, reproduction and use of the article provided the original source and authors are credited.

\section{Introduction}

Balance impairment due to diseases and deterioration of physiological systems is the main factor that causes the high risk of fall, especially in elderly adults, patients, and obese people [1,2]. Several studies indicate that the overweight population has poorer performance of balance functions, which results in the increased risk of falls. To reduce this risk, the Berg Balance Scale (BBS) is a popular tool for assessing people's balancing ability and predicting the risk of falling with high reliability [3, 4]. However, greater errors in balance evaluation may be found in overweight adults with lower ability of compensatory movements to maintain their postural steadiness [5]. Therefore, postural analysis with quantitative 
approaches, e.g. posturography, is considered to investigate balance impairment. In the case of posturography, center of pressure $(\mathrm{CoP})$ represents the resultant action of a vertical force on a surface $[6,7]$. CoP trajectories can reflect joint angles, angular velocities and accelerations to control the center of gravity $(\mathrm{CoG})$ over the base of support (BoS) [8]. These variables can be obtained by specific devices and computations including mathematical models.

Several studies focus on the mathematical models that reflect the balance ability of either healthy or balance impaired individuals. For normal balance ability, the postural control mechanism can be illustrated using different postural models. The single-link model is a simple approach to simulate the postural control mechanism in the anterior-posterior (AP) direction at the ankle joint [9, 10]. Angular velocity and acceleration are the primary factors that maintain the body's stability [11]. Additionally, these variables affect $\mathrm{CoG}$ positions due to the increase or decrease in plantar flexor activation. Moreover, the postural control mechanisms can describe the underlying multi-link models. For example, Koozekanani et al. [12] reported the biomechanical models to mimic postural control mechanism and define CoP positions in the AP direction.

In the case of postural control mechanism in the medio-lateral (ML) direction, a different method can be used to acquire the model. The relationship of contractions of two muscles, such as the gluteus medius and psoas major muscles, is an example of postural models in the ML direction and was derived by Jiang et al. [9]. Although these models were acquired by different methods, the outcomes from the models-such as torque of joint-are the primary variables to reflect the balance control mechanism. To complete the computation of the postural model, the kinematic data and specific variables such as angular displacement, body segment mass and length, and movement inertia are applied to estimate the torques and CoP positions in either the AP or ML direction. Based on these models, a wrong estimation of the anthropometric data negatively affects the simulation of the actual postural mechanism, especially in overweight cases. Therefore, a combination of soft computing technique and time series model is utilized to understand the postural control mechanisms in the overweight population using kinematic data. Fundamentally, there are several models of time series data; among them, the AR and MA models are widely used. The nonlinear autoregressive moving average (NARMA) model, which is a combination of these models, captures more complex structures of the time series [13].

To determine the coefficients of NARMA model, the Yule-Walker equation [14] and Burg's formulation [15] are generally applied in many articles. In this study, the artificial neural network (ANN) system is considered because this system is more effective in predicting nonlinear dynamic pattern, including system identification [16]. Therefore, the ANN system was used to investigate the difference in postural control mechanism between two groups-normal weight (NW) and overweight (OW) groups. The different mechanism in each group was represented in the form of the NARMA model, which was considered to define the postural model due to the relationship between body sway and CoP positions. This study investigated the following: (1) methods to determine the postural models underlying the neural network system and (2) methods to investigate the different postural control mechanisms between the NW and OW groups through the proposed technique. We expected that the difference in postural control mechanism could be identified using the proposed technique.

\section{Methodology}

\subsection{Participants}

Ten healthy adults were recruited from the community around Mahidol University. The inclusion criteria were as follows: (1) No history of neurological and/or musculoskeletal diseases that affect balance control, (2) no intake of medications that affect the balance control system, and (3) no consumption of alcohol for 12 hours before testing. Participants with a history of surgery and/ or orthopedic implants were excluded from the study. Prior to data collection, all the participants signed an informed consent that was approved by the Mahidol University Central Institutional Review Board (MU-IRB: 2014/112.1508). Demographic data and health information were obtained from each individual. Subsequently, these participants were categorized into the NW and OW groups, with body mass index (BMI) as the criterion. Participants with BMI between 20 to $24.9 \mathrm{~kg} / \mathrm{m}^{2}$ were assigned to the NW group, whereas participants with $\mathrm{BMI} \geq 25.0 \mathrm{~kg} / \mathrm{m}^{2}$ were grouped in the OW group [17]. The participant characteristics and balance performance for both groups are shown in Table 1. The results indicated significant differences between the NW and OW groups in weight and BMI.

\subsection{Data acquisition}

The postural steadiness of each individual was collected using two devices: a three-dimensional (3D) camera motion capture system and force plate. The OptiTrack motion capture system (Natural Point Inc., Oregon, USA) consists of eight infrared cameras (model s250e) with $832 \times 832$ pixels and requires markers to capture the motions. The Tracking Tools ${ }^{\circledR}$ version 2.5.0 software was used to capture and record real-time motion and export 
Table 1 Physical characteristics and balance performance of the participants in the normal weight (NW) and overweight (OW) groups. ${ }^{1}$

\begin{tabular}{|c|c|c|c|}
\hline \multirow{2}{*}{$\begin{array}{l}\text { Characteristics } \\
\text { of Participants }\end{array}$} & \multicolumn{2}{|c|}{ Mean (SD) } & \multirow[b]{2}{*}{$p$-value } \\
\hline & $\begin{array}{c}\text { NW } \\
(n=5)\end{array}$ & $\begin{array}{c}\text { OW } \\
(n=5)\end{array}$ & \\
\hline \multicolumn{4}{|c|}{ Physical Characteristic } \\
\hline Age (years) & $55.40(7.23)$ & $52.00(7.35)$ & 0.690 \\
\hline Height (m) & $1.60(0.03)$ & $1.57(0.09)$ & 0.841 \\
\hline Weight (kg) & $56.80(2.68)^{*}$ & $71.06(11.11)^{*}$ & 0.032 \\
\hline $\begin{array}{c}\text { Body Mass } \\
\text { Index }\left(\mathrm{kg} / \mathrm{m}^{2}\right)\end{array}$ & $22.27(1.41)^{*}$ & $28.54(2.25)^{*}$ & 0.008 \\
\hline \multicolumn{4}{|c|}{ Balance Performance } \\
\hline $\begin{array}{l}\text { CoP range in AP } \\
(\mathrm{mm})\end{array}$ & $13.43(5.60)$ & $18.08(8.92)$ & 0.251 \\
\hline $\begin{array}{l}\text { CoP range in ML } \\
(\mathrm{mm})\end{array}$ & $4.80(1.58)$ & $7.14(3.68)$ & 0.465 \\
\hline $\begin{array}{l}\text { Total excursion of } \\
\text { CoP in AP (mm) }\end{array}$ & $64.11(18.85)$ & $68.35(16.07)$ & 0.602 \\
\hline $\begin{array}{l}\text { Total excursion of } \\
\text { CoP in ML (mm) }\end{array}$ & $38.04(6.25)$ & 42.31(11.13) & 0.465 \\
\hline
\end{tabular}

the position of the markers. Six markers, $5 / 8$ inches in diameter, were attached to the $7^{\text {th }}$ cervical vertebra $(C 7)$, $4^{\text {th }}$ lumbar vertebra (L4), greater trochanter of the right and left femurs, and the lateral malleolus of both legs to receive the $3 \mathrm{D}$ coordinates and calculate the sway angle at the trunk and ankle joints. These coordinates were recorded in mm at a sampling rate of 64 frames per second.

A plantar pressure mat was used to simultaneously collect the CoP along the AP and ML directions. A MatScan ${ }^{\circledR}$ pressure mat model 3150 (TekScan Inc., South Boston, USA) was used in the study at a $64 \mathrm{~Hz}$ sampling rate. The Sway Analysis Module $\left(\mathrm{SAM}^{\mathrm{TM}}\right)$ is a software that connects to this pressure mat to export the sway data. While collecting data, the participant had to cross their arms and gaze at a red, rectangular reference point. This point was 2.5 meters away from the participant. The duration of data collection was two minutes, which corresponded to the direction of the BBS. Only one trial was conducted to collect data regarding the postural steadiness of each individual.

\subsection{Protocol of training and testing NN system}

The three main steps of training ANN systems-training, after training and testing processes- are shown in

\footnotetext{
${ }^{1}$ Note: Significant difference $(p$-value $<0.05)$ between groups is shown in bold number.
}

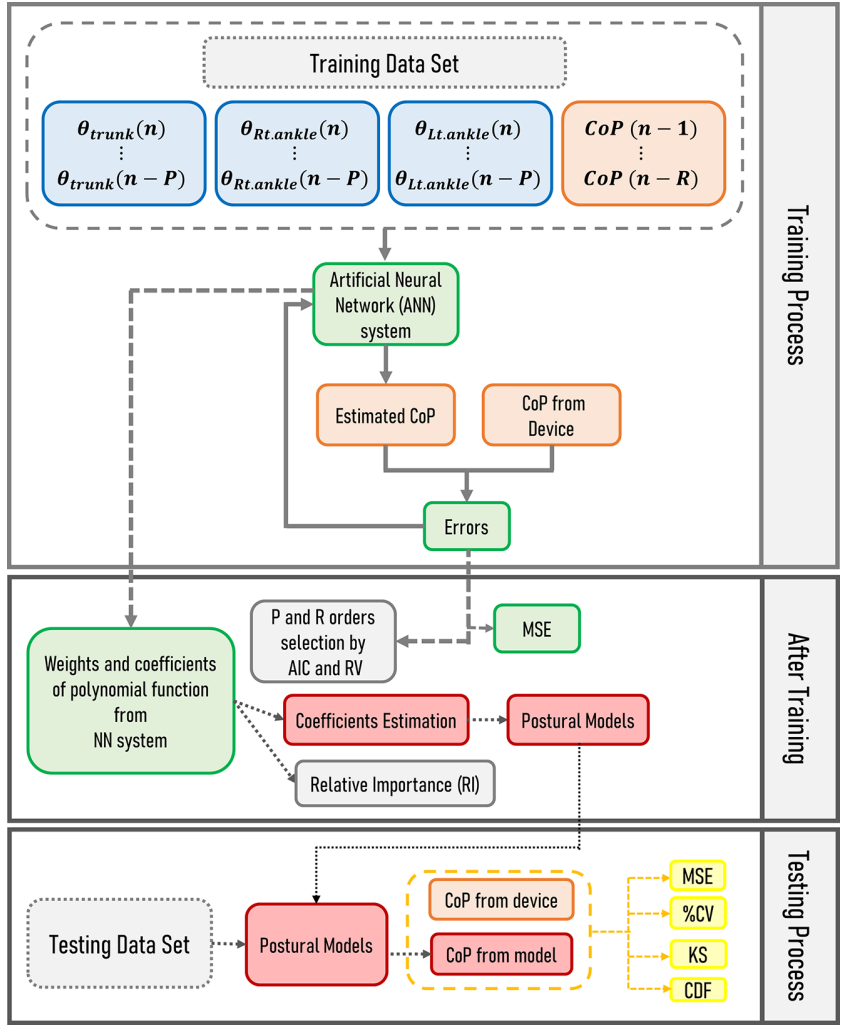

Fig. 1 Diagram of the training and testing processes to define the postural control model for one group.

Fig. 1. The training process comprises three main components-the angular displacements, CoP trajectories, and ANN system. This process commenced when the training data set in group A, B, C or D was applied to the ANN system. Group A and B represented the data sets from the NW group, whereas group C and D were the data sets in AP and ML directions, respectively, from the OW group. The outputs of the NN system were the CoP trajectories for each group, which were then compared to the $\mathrm{CoP}$ trajectories collected by the plantar pressure mat to provide the error values. These errors were used to update the weights while training the ANN system. When the error was less than 5\%, the training process would be stopped.

After completing this process, the updated weights, coefficients of polynomial function, and errors were used to define the postural models in each group. The errors were used to determine the moving average (MA), $P$, and autoregressive (AR), $R$, orders of the postural models underlying the NARMA model. Additionally, the mean square error (MSE) was calculated based on these errors. The updated weights and coefficients of the polynomial function of the ANN system were used to identify the postural model and its coefficients, including the relation between input and output of ANN system in each group. Ten trials were performed to construct the postural mod- 
el in each group.

The testing process was performed to define the postural model that could estimate the CoP trajectories similar to the CoP from the force plate. This process consisted of four predominant components_-angular displacements, CoP trajectories, postural models, and two variables for model evaluations - as shown in Fig. 1. This process began with the testing data sets in groups A, $\mathrm{B}, \mathrm{C}$ and $\mathrm{D}$ being applied to the postural model. The CoP trajectories were estimated through the postural models. To identify the postural model in each group, the MSE and percent coefficient of variation (\%CV), CDF plot and Kolmogorov-Smirnov (KS) test were considered.

\subsection{Data preprocessing}

The training and testing of data sets for the proposed system were performed using MATLAB version $2017 \mathrm{~b}$ (The MathWork Inc., Massachusetts, USA). Five participants in each group were randomly assigned to either the training or testing data set. The training data set was collected from three participants, while the postural steadiness data collected from the other two participants was established as the testing data set. The training and testing processes in each group comprised of two data sets. Each data set consisted of input and output data for determining the postural control model in either the AP or ML direction.

$3 \mathrm{D}$ coordinates on the trunk and both legs were considered to determine the angle displacements of the trunk and both ankle joints in the AP and ML directions. The process of data preprocessing was initiated by splitting the data into 30 -second intervals because a length of 30 seconds is recommended to assess the balance ability in adults [6]. Subsequently, these coordinates were subtracted from their means and filtered by the second order of the low-pass Butterworth filter with the cut-off frequency at $1 \mathrm{~Hz}$. Following this, the filtered data was used to compute the angular displacement in each direction. The angular displacements at the trunk and both ankle joints in the AP direction were determined by $\tan ^{-1}\left(\frac{y_{i}-y_{j}}{x_{i}-x_{j}}\right)$, where $x_{i}, x_{j}, y_{i}$ and $y_{j}$ are the $\mathrm{x}$ - and $\mathrm{y}$ coordinates of the proximal marker $i$ and distal marker $j$, respectively, in each body segment. In the ML direction, the angle displacements were calculated by $\tan ^{-1}\left(\frac{y_{i}-y_{j}}{z_{i}-z_{j}}\right)$, where $z_{i}$ and $z_{j}$ are the z-coordinates of the proximal and distal markers in each body's segment. Finally, the angular displacements of three joints were normalized from -1 to 1 . These variables were assigned as the input data $u(n)$.

The CoP trajectories on both directions were also split into 30-second intervals similar to the kinematic data. Subsequently, the data was subtracted by the CoP mean in each direction and filtered by the second order of the low-pass Butterworth filter with the cut-off frequency at $4 \mathrm{~Hz}$ for both directions. These filtered $\mathrm{CoP}$ signals were then normalized from -1 to 1 . The normalized signals were provided as the output data $y(n)$.

\subsection{Artificial Neural Network (ANN) system}

Generally, the ANN system is a simple parallel computational structure that consists of three layers: input, hidden, and output. The NARMA model in this study was modified from Chon and Cohen [18] and is denoted as follows:

$$
\begin{aligned}
y(n)= & \sum_{q=1}^{N=3} \sum_{j=0}^{P} a_{q}(j) u_{q}(n-j)+\sum_{k=1}^{R} b(k) y(n-k) \\
& +\sum_{q=1}^{N=3} \sum_{i=0}^{P} \sum_{j=0}^{P} a_{q}(i, j) u_{q}(n-i) u_{q}(n-j) \\
& +\sum_{k=1}^{R} \sum_{l=1}^{R} b(k, l) y(n-k) y(n-k) \\
& +\sum_{q=1}^{N} \sum_{j=0}^{P} \sum_{k=1}^{R} c(j, k) u_{q}(n-j) y(n-k) \\
& +\ldots+e(n)
\end{aligned}
$$

where $u$ and $y$ represent the input and output variables, respectively. The input variables consisted of previous and current data, while the output variables consisted of previous data. $q$ denotes the number of $q^{\text {th }}$ input variables from a training data set. $a_{q}(j)$ and $b(k)$ are the coefficients of the linear MA and AR terms. $a_{q}(i j), b(k, l)$ and $c(j, k)$ are the coefficients of nonlinear term in the model. $i, j, k$ and $l$ represent the indices on functions $u$ and $y$, respectively, and $e(n)$ is the error. From equation (1), this formula can be rewritten into the architecture of the ANN system, as shown in Fig. 2. The output from this architecture can be computed with the following equations:

$$
\begin{aligned}
& y(n)=\sum_{i=1}^{M} c_{i} p_{i}\left(x_{i}\right)+e(n) \\
& \text { where } p_{i}\left(x_{i}\right)=\sum_{s=0}^{1} a_{i s} x_{i}^{s} \\
& x_{i}=\sum_{q=1}^{N} \sum_{j=0}^{P} w_{q}(i, j) u_{q}(n-j)+\sum_{k=1}^{R} v(i, k) y(n-k)
\end{aligned}
$$

From equation (2), the output can be estimated from the summation of the polynomial function $p_{i}\left(x_{i}\right)$ and the weights at the hidden node of the $i^{\text {th }}$ and the output layers $c_{i}$. $M$ denotes the number of hidden nodes. Three components are involved in determining the polynomial func- 


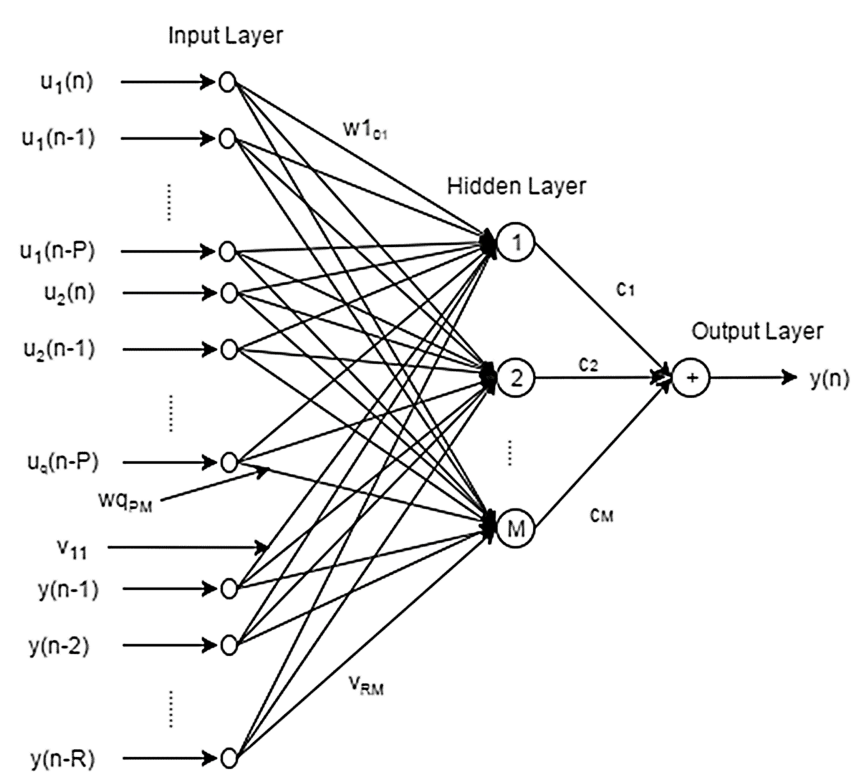

Fig. 2 Architecture of the ANN system to determine the coefficients of postural model in each group.

tion in each node, as shown in equation (3). First, $a_{i s}$ represents the coefficients' parameters that relate to the polynomial function in each hidden node. Second, $s$ is the order of the polynomial function and $x_{i}$ is the summation of input/output data and its weight. $x_{i}$ can be written as shown in equation (4). $w$ and $v$ are the weights between the input, output, and hidden node values. In this study, the weights between each layer were updated using the method of Aibinu et al. [19]. First, the updated weights on the output neuron were defined by the following:

$$
c_{i}^{\prime}=c_{i}+(\eta \delta) p_{i}
$$

where $\eta$ is the learning rate, and $\delta$ is the output error that is defined by the following:

$$
\delta(n)=t(n)-y(n)
$$

where $t(n)$ is the desired output. Second, the coefficient update in the polynomial function was performed with the following:

$$
a_{j i}^{\prime}=a_{j i}+\eta \delta_{1} \frac{\partial p_{i}}{\partial a_{j i}}
$$

where $\frac{\partial p_{i}}{\partial a_{r i}}=x_{i}^{r} ; r=0$ and 1 is a set that provides the same number of orders in the polynomial function. To complete equation (7), the back propagation of the error with weights in the output neuron was defined by multiplying the weights $c_{i}$ and error $\delta(n)$. This is shown in equation (8).

$$
\delta_{i}(n)=c_{i} * \delta(n)
$$

Third, the weights between the input and hidden layers were updated with these equations:

$$
w_{q}^{\prime}(i, j)=w_{q}(i, j)+\eta \delta_{i} \frac{\partial p_{i}(x)}{\partial(x)} u_{q}(n-j)
$$

$$
v^{\prime}(i, k)=v(i, k)+\mu \delta_{i} \frac{\partial p_{i}(x)}{\partial x} y(n-j)
$$

where $\frac{\partial p_{i}}{\partial x}=\sum_{k=1}^{2} k a_{k i} x^{(k-1)}$. After completing the training step, all the updated weights were used to determine the coefficients of the postural model, computed using the following equations:

$$
\begin{aligned}
& a_{q}(i)=\sum_{q=1}^{N} \sum_{s=1}^{M} c_{s} a_{q}(i s) w_{q}(i s) \\
& b(i)=\sum_{s=1}^{M} c_{s} a_{i s} v_{i s}
\end{aligned}
$$

The learning rate of the ANN system was 0.3 for all the groups. The number of input nodes was determined by the input data, output data, and $R$ and $P$ orders. The total input data $u$ in each model was the summation of three parameters multiplied by the $P$ order. For the total output data $y$, the number of previous $\mathrm{CoP}$ trajectories on either the AP or ML direction relied on the $R$ order. In the other layers, each hidden node was composed of the first order of the polynomial function, and the output layer consisted of one output node. The $P$ and $R$ orders, including the hidden nodes, were defined to acquire the accurate NARMA model with ANN [18]. According to these findings, the $P$ and $R$ orders rely on the ANN system during the training process. This is because these orders are used to identify the number of input nodes and sampling data. To determine the optimal orders, two methods of model selection-Akaike's information criteria (AIC) and residual variance (RV) criteria-were considered in the study.

\subsection{Selection of $\boldsymbol{P}$ and $\boldsymbol{R}$ orders}

AIC is a popular method for model selection [20, 21]. This is because it can accurately identify effective fitting models and reflect model complexity. To determine the order, the modified equation from Aibinu et al. [20] was used, as shown below:

$$
A I C=N \ln \left(\sigma_{P, R}^{2}\right)+2(P+R)
$$

where $N$ denotes the data length, $P$ and $R$ are the integral constant MA and AR orders, and $\sigma_{P, R}^{2}$ is the residual sum of squares (RSS) that is divided by the total amount of data length during training process. RSS is provided by the following:

$$
\sigma_{P, R}^{2}=\frac{1}{N} \sum_{i=K}^{N-1}\left(d_{i}-y_{i}\right)^{2} ; K=1,2,3, \ldots, N
$$

where $d_{i}$ and $y_{i}$ are the desired and estimated outputs at the $i^{\text {th }}$ sampling data, respectively. The equation proposed by Aibinu et al. [20] was used to determine the RV criteria, as given below. 


$$
R V(P, R)=\left(\frac{N-(P+R)}{N-2(P+R)-1}\right) \sigma_{P, R}^{2}
$$

The RV is based on the assumption that if the terms of the NARMA model do not fit comprehensively, the increase in the estimated variances will be obtained. The increase in these variances depends on the AR or MA terms in any model [20].

\subsection{Outcome measures}

\subsubsection{Minimum criteria values}

Minimum criteria values reflect the minimal values of AIC and RV. To determine these criteria, two steps were undertaken. First, the order model with $P, R=(2,1)$, $(3,2),(4,3), \ldots(8,7)$ was applied to define the criteria values. For the order of each pair, ten trials were repeated for $M=2,3, \ldots, 9$. Subsequently, the average criteria values for the order of each pair were compared to define the minimal criteria value. Second, the pair with the minimal criteria value was considered to identify the optimal $P$ and $R$ orders. In this case, the $P$ order and a number of hidden nodes were fixed. The $R$ orders, on the other hand, were adjusted from 1 to 10 . Ten trials were also conducted for the order of each pair to define the minimal criteria value. Finally, the averages of each pair were compared to investigate the optimal orders.

\subsubsection{CoP estimation by postural models with ANN system}

CoP estimation was achieved through equation (2) after the training process. To complete these models, the updated weights and coefficients of the polynomial function in the ANN system were used to identify the coefficients of postural model with equations (11) and (12). Following this, the estimated $\mathrm{CoP}$ positions were filtered with the second order of the Butterworth low-pass filter at the $4 \mathrm{~Hz}$ cut-off frequency and were normalized from -1 to 1 . Subsequently, the normalized CoP positions from the device and postural models were used to investigate the model performance.

\subsubsection{Mean square error (MSE)}

MSE was considered to investigate the performance of the postural models in each group during the training and testing processes because it is simple and effective in defining the loss function [22]. The formula for computing the error is indicated in equation (16). A low MSE implies that the model is accurate if the estimated output corresponds to the measured data from the device. In this case, a MSE $<5 \%$ was acceptable for the training and testing processes.

$$
M S E=\left(\frac{1}{N} \sum_{i=1}^{N}\left(d_{i}-y_{i}\right)^{2}\right) \times 100 \%
$$

\subsubsection{Percent of coefficient of variation $(\% \mathrm{CV})$}

Coefficient of variation $(\mathrm{CV})$ is one of the statistical measurements that reflects the data distribution around the median. Accordingly, a large CV indicates large data distribution that results in low precision of the model. Before defining the $\% \mathrm{CV}$, the CoP positions from the device and postural models were normalized from 0 to 1 . Subsequently, the normalized data was used to determine $\% \mathrm{CV}$ by using the following equation:

$$
\% C V=\left(\frac{I Q R}{\text { Median }}\right) \times 100
$$

where the interquartile range (IQR) was determined by the differentiation of the $75^{\text {th }}$ and $25^{\text {th }}$ quartile.

\subsubsection{Relative Importance (RI) of input neuron}

The relative importance (RI) was employed to determine the relationship between the input and output underlying the weights inside the NN system. For this study, Garson's algorithm (GA) was utilized to investigate which input or output variable in the input layer of the NN system affects CoP estimation in each group. The formula of GA is illustrated below.

$$
R I_{x}=\sum_{y=1}^{M} \frac{\left|w_{x y} \sum_{k=0}^{1} a_{k} w_{y z}\right|}{\sum_{x=1}^{n}\left|w_{x y} \sum_{k=0}^{1} a_{k} w_{y z}\right|}
$$

where $R I_{x}$ is the relative importance of input neuron at node $x^{\text {th }} ; w_{x y}$ and $w_{y z}$ are the connection weight between input node $x$ and hidden node $y$ and between hidden node $y$ and output node $z ; a_{k}$ is the coefficient of the polynomial function at $k^{\text {th }}$ order. The RI in each input node was used to rank from the highest to smallest order. The median in each variable in the input node was defined to distinguish the postural control mechanisms between two groups.

\subsection{Statistical analysis}

PASW Statistics 18.0.0 [SPSS (HongKong) Ltd., Quarry Bay, Hong Kong] was used for statistical analysis in this study. The KS test showed no normality in kinematic and CoP data. Therefore, a nonparametric statistical analysis was applied. The Mann-Whitney $U$ test was used to determine the difference between the physical characteristics of the NW and OW groups. To define the optimal postural model in the testing process, the MSE and \%CV were ranked from the smallest to highest order. Following this, the ranking of both the variables was defined with the following equation:

ranking $=\frac{\text { ranking }_{M S E}+\text { ranking }_{\% C V}}{2}$

The model that had the minimal ranking was as- 
signed as the postural model in each group. To investigate the performance of the proposed postural models, the KS test was performed to compare the outcomes from the postural models with the CoP positions from the device. The result in which the model was similar to the device showed high $p$-value null hypothesis $(H=0)$. In the case of rejection of the null hypothesis $(H=1)$, the output from the model was not the same as the device, and the $p$-value was small. Additionally, the CoP positions from the postural model of each group was compared based on the cumulative distribution function (CDF). The CDF plot illustrates the reliability of the model, underlying the cumulative probability associated with the function. The outcomes between the model that had maximum CDF distance from the device were different.

\section{Results}

The participant characteristics and postural performance in groups A, B, C and D are described in supplementary file 1. The findings revealed that there was no significant difference between training and testing data set for all groups. Additionally, the mean, standard deviation (SD) and $\% \mathrm{CV}$ of the training and testing data sets in each group were defined.

\section{1 $P$ and $R$ order selection for the postural models with ANN system}

Our results revealed that the minimum criteria values from the AIC and RV approaches were different in the two groups. For the first step of order selection, the results of the NW group showed that the minimum criteria values of the postural model in the AP direction were $P=$ $7, R=6$, and $M=9$. The values of the model in the ML direction were $P=2, R=1$, and $M=8$. The minimal AIC and RV values of the postural models were $-38,527.3$ and 0.001326, respectively, in the AP direction, while the criteria values of the models in the ML direction were $-35,514$ and 0.00227 . The MSE values of the two models were $0.132 \%$ and $0.226 \%$ in the AP and ML directions, respectively.

For the OW group, the minimum criteria values for the model in the AP direction were $P=2, R=1$, and $M=$ 9. The criteria values for the model in the ML direction were $P=5, R=4$, and $M=9$. In this case, the $P$ orders higher than 6 for the model in the AP direction and higher than 5 for the model in the ML direction were not observed, because large errors occurred during the training process of the ANN system. Thus, the criteria values were not defined. The AIC and RV criteria values of the models for the OW group were $-36,498$ and 0.001896 for the AP direction, while the criteria values on the ML direction were $-34,828.1$ and 0.002372 , respectively.
Table 2 Criteria values and MSE during the training process of the ANN system in the NW and OW groups.

\begin{tabular}{cccc}
\hline \hline \multicolumn{1}{c}{ Variables } & Direction & NW & OW \\
\hline Criteria values & & & \\
\multirow{2}{*}{ AIC } & AP & $-35,741.0$ & $-33,727.0$ \\
& ML & $-32,618.1$ & $-34,068.0$ \\
\multirow{2}{*}{ RV } & AP & 0.002042 & 0.002862 \\
& ML & 0.003486 & 0.002716 \\
Error value & & & \\
\multirow{2}{*}{ MSE } & AP & $0.204 \%$ & $0.286 \%$ \\
& ML & $0.348 \%$ & $0.271 \%$ \\
\hline
\end{tabular}

For the ANN training process error in the OW group, the MSE of the postural models in the AP and ML directions were $0.190 \%$ and $0.237 \%$, respectively.

To acquire the optimal $P$ and $R$ orders of the postural models in each group, the minimum criteria values were investigated when the $P$ order and a number of hidden nodes were fixed. For the NW group, the optimal orders of the postural model in the AP direction were $P=$ 7 and $R=9$. The order model in the ML direction were $P=2$ and $R=2$ (see supplementary file 2). In the OW group, the optimal orders of the postural model in the AP direction were $P=2$ and $R=4$. The order model in another direction were $P=5$ and $R=3$ (see supplementary file 3). The AIC, RV, and their errors due to the training process in each group are shown in Table 2.

In the case of the sampling data, the number of input data for the training and testing processes depended on the input nodes which relayed the $P$ and $R$ orders. Thus, the data for the training and testing processes for all the groups were defined by the following equation:

$$
\begin{aligned}
S_{\text {data }}=[ & {\left[\left(L_{\text {data }}-\max _{\text {Order }}\right) \times((\text { input } \times P)\right.} \\
& +(\text { output } \times R))] \times n_{p}
\end{aligned}
$$

where $S_{\text {data }}$ is the number of sampling data in each group; $L_{\text {data }}$ represents the total frames, which is 1,920 frames per participant; max $_{\text {Order }}$ is the maximal $P$ or $R$ orders in each group; input and output are the number of considerable variables in this study; and $n_{p}$ is the number of participants assigned in the training and testing processes. Accordingly, the total sampling data from the training and testing processes are illustrated in Table 3.

\subsection{Defining the postural models with the ANN sys- tem}

The testing data set was employed to define the optimal postural model. The results revealed that the postural models provided the CoP trajectories, which were simi- 


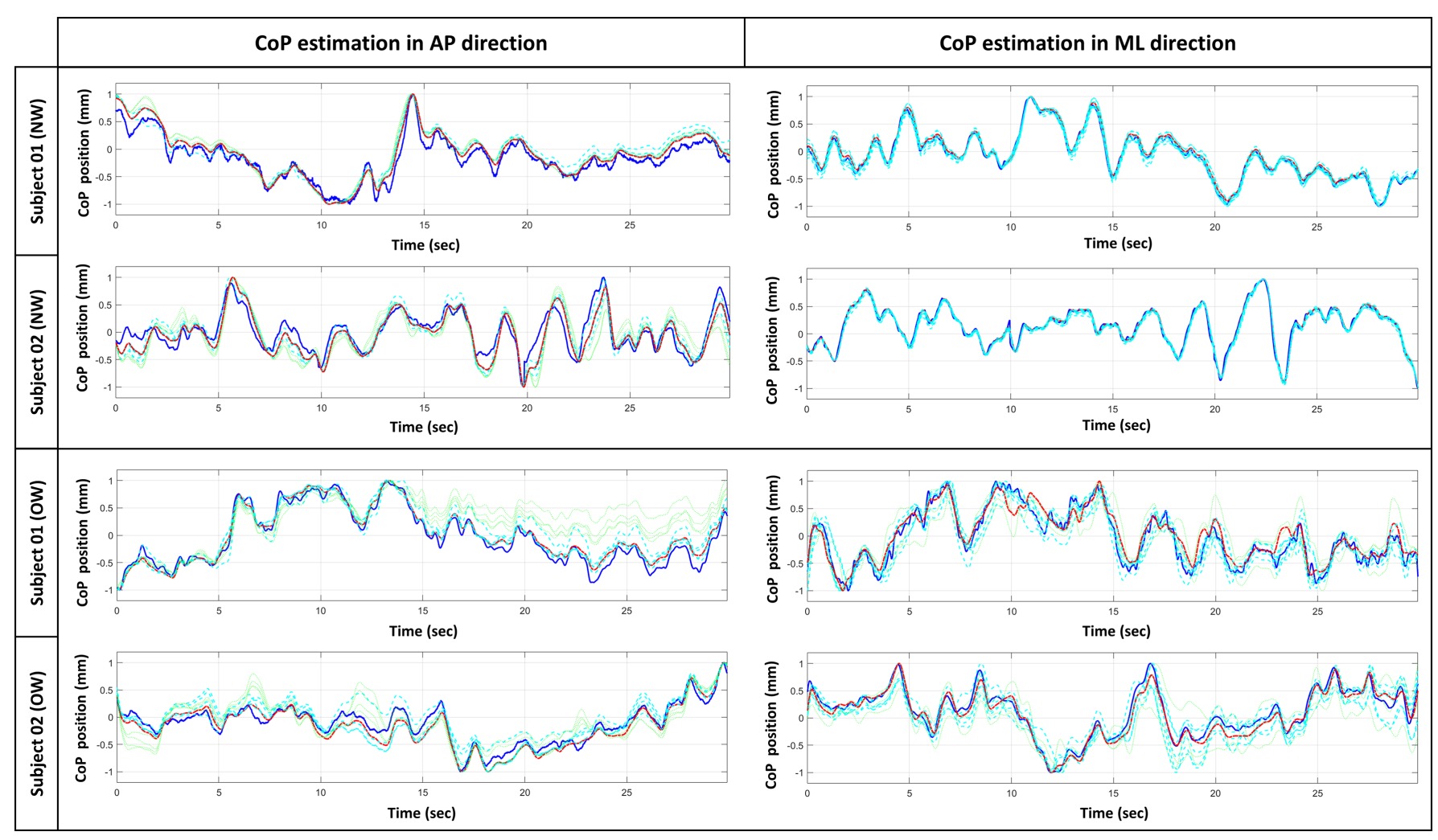

Fig. 3 The CoP positions from the device (blue solid line) and postural models with the ANN system (dash line) for each group.

Table 3 Sampling data during the training and testing processes of the ANN system in the NW and OW groups.

\begin{tabular}{|c|c|c|c|}
\hline Variables & Direction & NW & OW \\
\hline \multicolumn{4}{|c|}{ Sampling data (training process) } \\
\hline \multirow{2}{*}{$S_{\text {data }}$} & $\mathrm{AP}$ & 171,990 & 57,480 \\
\hline & ML & 46,032 & 103,410 \\
\hline \multicolumn{4}{|c|}{ Sampling data (testing process) } \\
\hline \multirow{2}{*}{$S_{\text {data }}$} & AP & 114,660 & 38,320 \\
\hline & ML & 30,688 & 68,940 \\
\hline
\end{tabular}

lar to the CoP positions from the force plate ${ }^{2}$ (see Fig. 3). To determine the optimal model, MSE and \%CV were considered. Our results revealed that the postural models with MSE $<5 \%$ in each group were provided by the ANN system, especially the ML direction model in the $\mathrm{NW}$ group. Interestingly, the results revealed that $\% \mathrm{CV}$ values of the models with $\mathrm{MSE}<5 \%$ were similar to the $\% \mathrm{CV}$ of CoP positions from the device (see supplementary file 4). Although the output from these models are

\footnotetext{
${ }^{2}$ Note: The green and light blue lines indicate the CoP estimations with MSE $>5 \%$ and $<5 \%$, respectively. The red line shows the $\mathrm{CoP}$ estimation with the lowest MSE and \%CV.
}

similar to the device (see Fig. 3), the models with the minimal rankings of both variables were assigned as the postural control systems for each group. The MSE, \%CV of CoP estimation and CDF plot of the postural model with the minimum ranking in each group are illustrated in Table 4 and Fig. 4.

As shown in Fig. 4, the results revealed that the CoP estimation from the postural models is similar to the CoP trajectories from the device. This was because the CDF plot from the model and device was no difference. Additionally, the maximum distances of the CDF plot between the postural models and device were less than 0.12 for all groups. However, our findings from the statistical analysis investigated that the CoP positions estimated by the postural model in the ML direction for one from two participants of the NW group were congruent to those estimated by the device $(H=0, p$-value $=0.743)$, which corresponded to the smallest CDF distance in both participants (see Table 4). Although the estimated CoP positions in the ML direction from the postural model were similar to the CoP positions from the device, there was no significant correlation in another participant from the same group $(H=1, p$-value $=0.013)$. Moreover, the results revealed that the postural models in the other group could not estimate CoP positions similar to the ones estimated by the device $(H=1, p$-value $<0.001)$. 

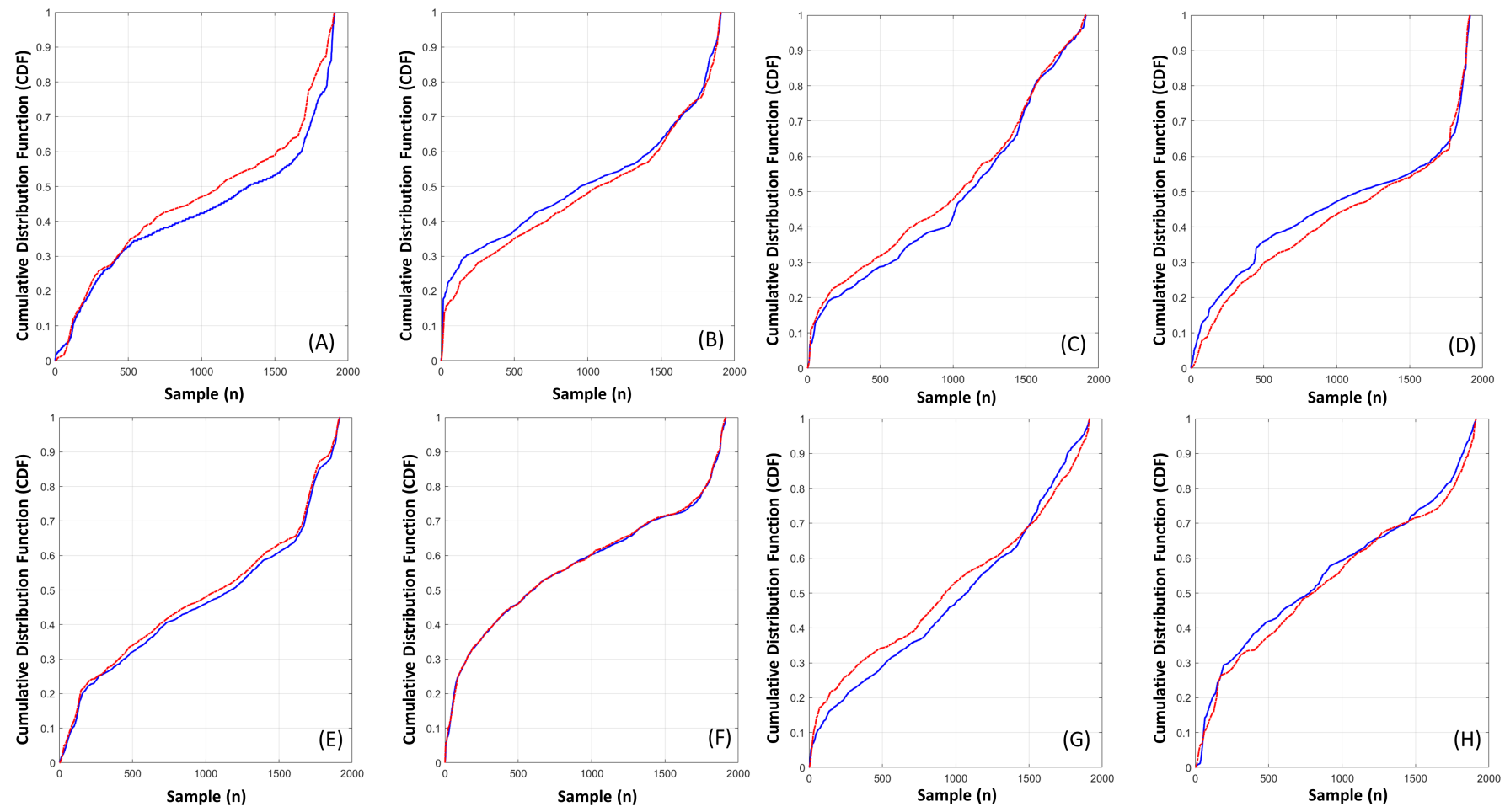

Fig. 4 The cumulative distribution function (CDF) plot which compares the CoP positions from the device (blue solid line) with the postural models of the ANN system (red dot line). (A)-(B) and (E)-(F) represent the model in AP and ML directions for the NW group. (C)-(D) and (G)-(H) represent the model in the AP and ML directions for the OW group.

Table 4 Mean square error (MSE), percent of coefficient of variation $(\% \mathrm{CV})$, and maximal distance of the cumulative distribution function (CDF) of CoP estimation from the postural models for each group.

\begin{tabular}{ccccc}
\hline \hline \multirow{2}{*}{ Variables } & \multicolumn{2}{c}{ NW group } & \multicolumn{2}{c}{ OW group } \\
\cline { 2 - 5 } & AP & ML & AP & ML \\
\hline All participants & & & & \\
MSE & $2.40 \%$ & $0.24 \%$ & $1.33 \%$ & $2.13 \%$ \\
\%CV & 51.46 & 50.52 & 63.43 & 64.42 \\
\hline Subject 01 & & & & \\
MSE & $1.49 \%$ & $0.29 \%$ & $1.18 \%$ & $3.09 \%$ \\
\%CV & 46.98 & 59.69 & 81.10 & 62.97 \\
CDF & 0.11 & 0.03 & 0.06 & 0.06 \\
Maximal distance of & & & & \\
\hline Subject 02 & & & & \\
MSE & $3.31 \%$ & $\mathbf{0 . 2 0} \%$ & $1.48 \%$ & $1.17 \%$ \\
\%CV & 51.26 & $\mathbf{4 2 . 9 4}$ & 57.18 & 60.43 \\
CDF & 0.10 & $\mathbf{0 . 0 3}$ & 0.07 & 0.05 \\
\hline \hline
\end{tabular}

\subsection{The relative importance (RI) of four variables to estimate the $\mathrm{CoP}$ positions}

Our findings revealed that the kinematic data and $\mathrm{CoP}$ positions affected $\mathrm{CoP}$ estimation by the $\mathrm{NN}$ system (see
Fig. 5). In the NW group, the results indicated that the $\mathrm{CoP}$ trajectories in the AP and ML directions had the smallest ranking of RI. On the contrary, the left and right ankle joints had the smallest ranking of RI for the ANN system to determine the postural model in the AP and ML directions, respectively. Interestingly, the results indicated that the dominant leg was used to maintain the equilibrium by different mechanisms.

\section{Discussion}

This is the first preliminary study that focuses on the application of the artificial neural network system for describing the postural control mechanism in overweight adults. Several studies indicate that the overweight population has poorer performance of the balance functions, which results in the increased risk of falls in the future. For this population, balance evaluation is therefore instrumental for reducing the incidence of falls. To evaluate balance impairment, several modalities have been examined, such as clinical balance assessment and equipment used. However, several studies have revealed that there is no significant difference between the balance performance of the NW and OW groups [2]. Based on these reasons, other postural analysis methods were considered. In this study, the weights and coefficients in the polynomial function are valuable to clarify which input variable influences the CoP estimation, which can 


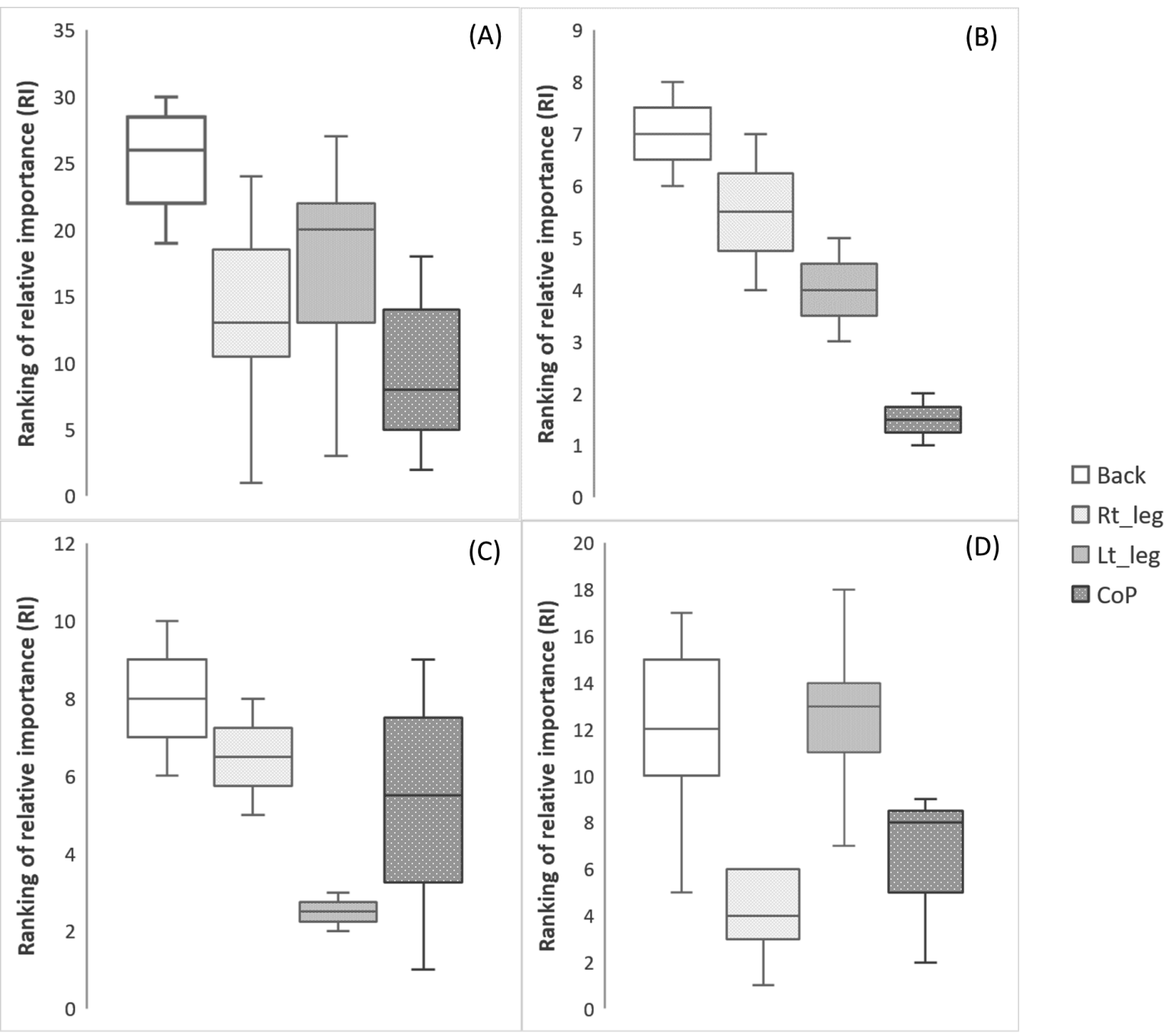

Fig. 5 Ranking of relative importance (RI) from four variables when (A) and (C) represent the results of postural models in the AP directions and (B) and (D) are the ranking of postural models in the ML direction from NW and OW groups, respectively.

reflect the different strategies to maintain equilibrium in each group. Additionally, the models derived by the proposed technique could project the CoP positions in each group.

Several functions were utilized to estimate the output from the data set. In this study, the recursive function is the basis of the proposed ANN system. In the training process, the kinematic data (input variable) and CoP positions (output variable) were used to define the optimal weights and coefficients of the polynomial function in the ANN system. After completing the training process, these values were utilized to define the postural model, including the relationship between the input and output of the ANN system. To obtain the postural model in each group, MSE, AIC and RV were observed to identify the $P$ and $R$ orders, including the number of hidden nodes $M$. Our results revealed that there were variations in optimal order for each group. However, the criteria values were not observed when the $P$ orders were larger than 6 or 5 for the models in the AP or ML directions, respectively. Since the \%CV of CoP positions in the NW group was higher than in the OW group for both directions (see supplementary file 1), this resulted in greater variation of CoP positions in the OW participants. Thus, the variation of data set caused overfitting of NN system when higher orders were observed.

Although the results showed that the postural models could estimate the CoP positions for both groups, it remained unknown which postural model was optimal for simulation in postural control mechanism. Accordingly, the MSE, AIC, and RV values were observed during training process to identify the orders for postural models. Our findings indicated that the MSE of the postural models in the training process was less than $5 \%$. Thus, the minimal criteria values were observed to identify the optimal orders for the postural model in each 
group. From our results, the minimum MSE, AIC, and $\mathrm{RV}$ values were applied to develop the most efficient ANN system, which resulted in different forms of postural control mechanism for each group.

During the testing process, there were ten postural models for each group. To choose the optimal postural model, the MSE and \%CV values were observed. Our findings revealed that the MSE of the postural model was less than 5\%, especially in the case of the ML direction model of the NW group. Additionally, the MSE, which was less than $5 \%$, had the same trend of $\% \mathrm{CV}$ as the device. Thus, the CoP estimation of those models was similar to the CoP measurement from the device. Based on these findings, MSE and $\% \mathrm{CV}$ were the criteria for choosing the postural model in each group.

In the case of model performance, the results of KS and CDF plot indicated that the models with minimum ranking of \% CV and MSE could estimate the output with greater accuracy and precision. We found no differences in outcome between the device and postural models, especially in the ML direction for the NW group (see Fig. 4). This was because there was a strong significant correlation in CoP position between the postural model and device. Furthermore, the MSE and \%CV values were lower in this model than the others. Additionally, the maximal CDF distance between the postural model and device was smaller than the other models, consistent with these findings. For the other groups, the postural models could estimate CoP positions correctly in some participants. We found that these models had larger MSE, \% CV, and maximal distance of $\mathrm{CDF}$, consistent with the statistical results.

For this study, the $P$ and $R$ orders depended on the training process of the ANN system, including the number of input and output variables. Interestingly, we found that there were differences in the number of orders for all the groups. Our results also revealed differences in the postural control strategies for both groups. For the NW group, the postural control mechanism relayed the information in the AP direction rather than in the ML direction. This was because the MA, $P$, and AR, $R$ orders of the model in the AP direction were larger. Interestingly, these models also illustrated the postural control strategies in each direction, since the $P$ and $R$ orders were used to assign the number of input and output data elements, respectively (see equation 1 ). In this case, the input data of the postural models in the AP and ML directions were 7 and 2 for each input variable, and the output data were 9 and 2. According to this, the postural control mechanisms in both directions did not correspond to the stimulus-response relation because the number of output elements was higher than the input. Based on this structure, the total input data were 21 and 6 for the AP and ML di- rections. The total input nodes of the models in the AP and ML directions were 30 and 8, respectively. This was due to the $R$ order being higher than the $P$ order, which corresponded to anticipatory postural adjustment. Anticipatory postural adjustment activates muscle contraction before a person's movement is initiated. This action could provide equilibrium due to muscle contraction. Furthermore, the postural control in the NW group also depended on the CoP trajectories rather than the body sway, especially in the AP direction. To control the equilibrium in this situation, the predicted displacements of the CoM played a role in the postural control rather than in the responses by afferent information [23].

The mechanism of postural control in the OW group was different from that in the NW group. The findings revealed that the highest $P$ order for the postural model was in the ML direction. Angular displacement was necessary to maintain an upright position in the ML direction, since the input data for the model in the AP and ML directions were 2 and 5 for each input variable while the output data in both directions were 4 and 3 . The total input data for both directions were 6 and 15, respectively. The total input nodes for the ANN system of the OW group were 10 and 18 . However, we observed anticipatory postural adjustment for both directions.

To perform an in-depth analysis of postural control mechanism, the weights and coefficients in the polynomial function were utilized to determine the relationship between the input and output of the ANN system. The findings revealed that the $\mathrm{CoP}$ positions in the $\mathrm{AP}$ and ML directions influenced CoP estimation for the NW group because this was the smallest ranking of RI. On the contrary, the kinematic data depended on the CoP estimation in the OW group (see Fig. 5). Interestingly, the results revealed that there were differences between the NW and OW groups in postural control mechanisms. The dominant leg of the NW group contributed to the postural control mechanism in the AP direction, while the non-dominant leg was utilized to maintain the equilibrium in the ML direction. On the contrary, the dominant leg of the OW group was used to maintain stability in the ML direction.

Several factors resulted in the increase of postural sway, especially in the ML direction, in the OW group. Weight gain is one of many factors that influence the changes in the body's structure and balance control mechanism. This is because weight gain induces greater plantar contact and pressure on the feet, particularly in the heel, midfoot, and metatarsal head. These regions contain mechanoreceptors that are vital to the balance control system. High pressure on the feet can reduce the reliability of afferent information from the mechanoreceptors because of a prolonged supra-threshold stimula- 
tion [24, 25]. Furthermore, weight gain increases inertia and holds the whole body in the stability zone for a shorter period [2]. Increased abdominal fat is related to high anterior tilt in overweight people. The structural change encourages increased pressure on the heels and compensatory movements in the balance function through forward shifting of the CoG position $[1,25]$. Our results can be summarized as follows. Maintaining an upright position is instrumental for the OW group to compensate for the balance function, especially in the ML direction. Based on these findings, the ANN system can investigate the differences in the postural control mechanisms between the NW and OW groups. Several studies have found no significant difference in the balance functions between the two groups. Furthermore, the proposed technique can provide the postural model that simulates a person's equilibrium using only the kinematic and CoP data. Although this study revealed that the proposed models could indicate the different balance strategies between the NW and OW groups, it had some limitations. First, the small sample size resulted in incorrect CoP estimation in some cases. Second, minor factors such as the distance between two feet were not controlled during data collection, which affected output estimation of the neural network system. Third, the proposed models could only be applied to describe the mechanism of human bipedal postural steadiness when the participants' eyes are open because the training data set was collected under this condition. Last, the input data depended on the $P$ order for all the input variables; the optimal $P$ order may vary in each parameter. Therefore, increasing the sample size would improve the accuracy of the postural models.

\section{Conclusion}

Our findings show the application of the artificial neural network system to define the postural control mechanism for overweight adults. Additionally, the results indicate that the proposed postural model can estimate the CoP positions with higher accuracy and precision. Furthermore, the findings reveal that there are differences in postural control strategy between the two groups. The postural control mechanism in the AP direction is necessary to maintain the upright position in the NW group. Conversely, the OW group relies on balance control in the ML direction. Interestingly, our findings indicate that both groups can maintain the upright position by utilizing the anticipatory postural adjustment.

\section{Acknowledgement}

The authors would like to thank all the participants for taking part in the balance assessment session.

\section{References}

1. Del Porto H, Pechak C, Smith D, Reed-Jones R: Biomechanical effects of obesity on balance. Int J Exerc Sci. 5(4), 301-320, 2012.

2. Dutil M, Handrigan GA, Corbeil P, Cantin V, Simoneau M, Teasdale N, Hue O: The impact of obesity on balance control in community-dwelling older women. Age. 35(3), 883-890, 2013.

3. Persad CC, Cook S, Giordani B: Assessing falls in the elderly: should we use simple screening tests or a comprehensive fall risk evaluation? Eur J Phys Rehabil Med. 46(2), 249-259, 2010.

4. Santos GM, Souza ACS, Virtuoso JF, Tavares GMS, Mazo GZ: Predictive values at risk of falling in physically active and no active elderly with Berg Balance Scale. Braz J Phys Ther. 15, 95-101, 2011.

5. Rogers ME, Page P, Takeshima N: Balance training for the older athlete. Int J Sports Phys Ther. 8(4), 517-530, 2013.

6. Duarte M, Freitas SM: Revision of posturography based on force plate for balance evaluation. Rev Bras Fisioter. 14(3), 183-192, 2010.

7. Palmieri RM, Ingersoll CD, Stone MB, Krause BA: Centerof-Pressure parameters used in the assessment of postural control. J Sport Rehabil. 11(1), 51-66, 2002.

8. Sasagawa S, Ushiyama J, Kouzaki M, Kanehisa H: Effect of the hip motion on the body kinematics in the sagittal plane during human quiet standing. Neurosci Lett. 450(1), 27-31, 2009.

9. Jiang Y, Nagasaki S, You M, Zhou J: Dynamic studies on human body sway by using a simple model with special concerns on the pelvic and muscle roles. Asian J Control. 8(3), 297-306, 2006.

10. Sovol AW, Bustamante Valles KD, Riedel SA, Harris GF: Bi-planar postural stability model: fitting model parameters to patient data automatically. Conference proceedings: Annual International Conference of the IEEE Engineering in Medicine and Biology Society. pp. 3962-3965, 2010.

11. Winter DA: Human balance and posture control during standing and walking. Gait Posture. 3(4), 193-214, 1995.

12. Koozekanani SH, Stockwell CW, McGhee RB, Firoozmand F: On the role of dynamic models in quantitative posturography. IEEE Trans Biomed Eng. 27(10), 605-609, 1980.

13. Chan NH: Autoregressive moving average models. In: Time series: applications to finance with $\mathrm{R}$ and S-Plus. 2 edition ed, John Wiley and Sons Ltd, pp. 23-37, 2011.

14. Adhikari R, Agrawl RK. An Introductory Study on Time Series Modeling and Forecasting. <https://arxiv.org/ftp/arxiv/papers/ 1302/1302.6613.pdf. $>$ [accessed on February, 2013].

15. Tanaka H, Nakashizuka M, Uetake T, Itoh T: The effects of visual input on postural control mechanisms: an analysis of center-of-pressure trajectories using the auto-regressive model. J Hum Ergol. 29(1-2), 15-25, 2000.

16. Jiang Y, Yang C, Na J, Li G, Li Y, Zhong J: A brief review of neural networks based learning and control and their applications for robots. Complexity. 2017, 1-14, 2017.

17. Nuttall FQ: Body mass index: obesity, BMI, and health a critical review. Nutr Today. 50(3), 117-128, 2015.

18. Chon KH, Cohen RJ: Linear and nonlinear ARMA model parameter estimation using an artificial neural network. IEEE Trans Biomed Eng. 44(3), 168-174, 1997.

19. Aibinu AM, Salami MJE, Shafie AA, Najeeb AR: Comparing au- 
toregressive moving average (ARMA) coefficients determination using artificial neural networks with other techniques. Int J Comput Inf Eng. 18, 1846-1851, 2008.

20. Aibinu AM, Najeeb AR, Salami MJE, Shafie AA: Optimal Model Order Selection for Transient Error Autoregressive Moving Average (TERA) MRI Reconstruction Method. Int J Comput Inf Eng. 18, 1834-1838, 2008.

21. Boardman A, Schlindwein FS, Rocha AP, Leite A: A study on the optimum order of autoregressive models for heart rate variability. Physiol Meas. 23(2), 325-336, 2002.

22. Rosenberg M, Thornton AL, Lay BS, Ward B, Nathan D, Hunt D, Braham R: Development of a Kinect software tool to classify movements during active video gaming. PLOS ONE. 11(7), 1-14, 2016.

23. Enoka RM: Automatic Responses. In: Neuromechanics of human movement. 4 ed., Human Kinetics, USA, pp. 272-288, 2008.

24. Ku PX, Abu Osman NA, Yusof A, Wan Abas WA: Biomechanical evaluation of the relationship between postural control and body mass index. J Biomech. 45(9), 1638-1642, 2012.

25. Son SM: Influence of obesity on postural stability in young adults. Osong Public Health Res Perspect. 7(6), 378-381, 2016.

\section{Thunyanoot Prasertsakul}

Ms. Thunyanoot PrasertsakUl received her B.Sc. in Physical Therapy from Mahidol University, Nakorn Phathom, Thailand in 2006 and M.Eng. in Biomedical Engineering from Mahidol University in 2010. She is currently a PhD student in Biomedical Engineering, Mahidol University. Her research

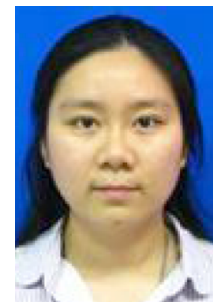
interests include rehabilitation engineering, human motion analysis and biomedical signal processing.

\section{Warakorn Charoensuk}

Dr. Warakorn Charoensuk received the B.E. degree in control engineering from King Mongkut's Institute of Technology, Ladkrabang, Thailand, in 1991 and the M.S. and Ph.D. degrees in electrical engineering from Vanderbilt University, Nashville, TN, in 1998 and 2001, respectively. Currently, he

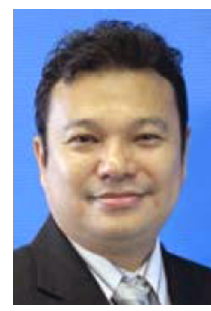
is an assistant professor at Biomedical Engineering Department, Faculty of Engineering, Mahidol University, Thailand. He serves in various national and international panels related to human motion analysis and development of sensor technology for sport. His main research interests are in wireless sensor in sport technology, biomedical signal processing, physiological system modeling and identification, human motion analysis and rehabilitation technology, and development of prostheses and orthoses for rehabilitation. 